\title{
Numerical study of the churn-slug transition dynamics in vertical upward air-water flows
}

\author{
F. H. J. Imada, F. Saltara \& J. L. Baliño \\ Departamento de Engenharia Mecânica, Escola Politécnica, \\ Universidade de São Paulo, São Paulo, Brazil
}

\begin{abstract}
In gas-liquid two-phase flows through vertical ducts, the spatial distribution of phases may assume several configurations, known as flow patterns. Slug and churn patterns are characterized by an unsteady behaviour and their boundary is a subject of interest for many researchers. Different descriptions of mechanisms occurring at churn/slug transition may be found in the literature. One transition process is described by Taitel et al. (Modelling flow pattern transitions for steady upward gas-liquid flow in vertical tubes. AIChE Journal, 26, pp. 345-354, 1980), in which churn flow is an entrance condition observed upstream of a developed slug flow if there is sufficient pipe length for this to happen. In the present work, vertical upward air-water flows through a 2 -inch i.d. tube are simulated with the commercial finite-volume-based code FLUENT, aiming to study this churn/slug transition process through spatial and temporal phase distribution visualizations and statistical analyses of void fraction histories at points along the domain. The incompressible Volume of Fluid (VOF) formulation allied to a Piecewise Linear Interface Construction (PLIC) technique is applied to capture the distribution of phases throughout three-dimensional domains. Turbulence effects are accounted through the Realizable k-epsilon model. Three different tube lengths and two sets of superficial velocities are studied in order to understand their influence in liquid slug collapse and bubble coalescence processes. Void fraction histories at points distributed along the duct centerline are stored during a simulation time long enough to provide statistical data with reasonable errors. Fast Fourier Transform analyses are used to verify the transition point and flow characteristic frequencies. Probability distributions of void fraction at cross sections along the tube are also evaluated. The VOF method was able to qualitatively capture the mechanisms of churn/slug transition processes. Results for entrance length and frequencies are
\end{abstract}


compared to correlations available in the literature. Probability distributions of void fractions are in good agreement with experimental data.

Keywords: multiphase flow, slug flow, churn/slug transition, computational fluid dynamics, volume of fluid.

\section{Introduction}

The study of gas-liquid two-phase flows receives great attention due to its occurrence in a wide range of industrial applications such as bubble column reactors in chemical and pharmaceutical industries, pressurized water reactors in nuclear plants, oil and gas extraction wells and transportation ducts. In vertical upward circular cross-section tubes, depending on operation conditions and fluid properties, the gas-liquid mixture may assume distinct topological configurations, i.e., the phases assume different spatial distributions within the domain, which are known as flow patterns. In general, vertical gas-liquid flows are classified as bubble, finely dispersed bubble, slug, churn or annular flow, each one presenting particular behaviour and hydrodynamic characteristics.

Such regimes may be identified through two different approaches [2]: regime maps elaborated with extensive experimental data or theoretical modeling of the pattern transition boundaries. Spedding et al. [2] summarized a collection of regime maps available in the literature. They indicated that different sets of mapping parameters are used and it is unlikely that one of them could be used in a universal map. Own experimental data were compared to such maps and two new relations for slug-churn and churn-annular transitions were suggested.

Through pattern transition modeling approach, researchers suggested mechanisms acting in the flow that lead to a transition. Particularly on churn-slug transition process, three different research lines may be presented. Nicklin and Davidson (apud. [3]) stated that this transition occurs due to flooding phenomenon in the liquid film surrounding the Taylor Bubble. Taitel et al. [1] suggested that churn flow is an entry region phenomenon upstream of a developed slug flow if there is enough pipe length for the transition to take place. Observing a fixed location in a tube, Mishima and Ishii [4] and Brauner and Barnea [5] indicated that the main mechanism acting in the destruction of a well-organized slug flow and leading to the transition towards a churn pattern is the excessive aeration of the liquid slug; increasing the gas flowrate, the gas void fraction within the liquid packet reaches the maximum bubble volumetric packing and a local concentration of small bubbles results in the destruction of the liquid bridge with consequential oscillatory motion. This approach is coherent with Taitel et al observations as, at a fixed position where slug flow is present, increasing the gas flowrate will also increase the entrance length, shifting the transition front to the location under study.

Theoretical models were also developed to estimate hydrodynamic characteristics of slug flow, such as bubble rise velocities and pressure drops. Bendiksen [6] developed an analytical expression for bubble rise velocity in stagnant fluid considering the effects of surface tension. He numerically calculated the bubble rise velocity in flowing liquid under influence of both laminar and turbulent velocity 
profiles. The results were used to provide approximated expressions for bubble rise velocity. Usual hydrodynamic models consider the bubble as a cylindrical shape with flat nose. This simplification was overcome by Barnea [7], who considered the curved nose shape in order to calculate the pressure gradient in vertical airwater slug flows. He compared his model with flat-nose ones and indicate that the neglect of nose curvature may lead to erroneous results.

Hydrodynamic characteristics of slug flow are also extensively studied through experimental methods. Jones [8] evaluated void fraction of air-water flows in rectangular ducts through X-ray measurements. He suggested the application of probability density functions (PDF) of void fraction for identifying the flow patterns and concluded that slug flow is a combination of bubble and annular regimes. Van den Akker et al. [9] applied pressure transducers and light attenuation probes along a vertical tube to capture slugging frequencies of air-water flows. They also measured Taylor bubbles and voidage waves propagation velocities, as well as bubble and liquid slug lengths. Their results led to a modification of the slugging frequency correlation proposed by Heywood and Richardson [10] originally developed for horizontal flows.

With impedance probes, Watson and Hewitt [3] measured the void fraction of air-water flowing in a $32 \mathrm{~mm}$ i.d., $12.6 \mathrm{~m}$ long tube for a variety of gas flowrates and operational pressures. Using the result histograms, they identified the flow patterns and made a comparison with theoretical models of transition boundaries. They described the typical histogram of a slug flow as having two peaks: one at low void fraction and another at high void fraction. The transition to churn flow is assumed when void fraction values are above 0.37. Polonsky et al. [11] studied the motion of a single Taylor bubble in both stagnant and flowing liquid. Through sequences of video images, they captured the instantaneous bubble shape and its evolution, also calculating its rise velocity. They evaluated the influence of gas compressibility by verifying the difference between bubble nose and tail velocities; they also observed the oscillatory motion of the bubble tail.

Van Hout et al. [12] used optical fiber probes for measuring instantaneous and average velocities and lengths of Taylor bubbles and water slugs in $24 \mathrm{~mm}$ and $54 \mathrm{~mm}$ i.d., $10 \mathrm{~m}$ long tubes with inclination angles in the range $2^{\circ}-90^{\circ}$. They also provided results for the evolution of slugging frequencies as the flow moves downstream. Additionally, Shemer [13] used particle image velocimetry (PIV) to capture the motion of the liquid film around the bubbles, obtaining velocity vectors in the bubble nose and tail regions. He also showed the evolution of liquid velocity in the wake region. Hernandez-Perez et al. [14] measured slugging frequencies of air-water flows in $38 \mathrm{~mm}$ and $67 \mathrm{~mm}$ i.d., $6 \mathrm{~m}$ long tubes and $0^{\circ}-90^{\circ}$ inclination angle range. Through the results, a correlation for slugging frequency was proposed.

With the advance in digital computers, numerical simulations of two-phase flows are also being performed. Basically, three Eulerian modeling approaches are available.

The most complete approach is the Separated Phases modeling, commonly known as Two-Fluid model, in which separate conservation equations are solved 
for each phase. Ekambara et al. [15] applied the Two-Fluid model to simulate horizontal air-water bubbly flows at different inlet superficial velocities and air volume fractions. Results of water velocity and void fraction profiles obtained with two different turbulence models were compared to experimental data. The interfacial forces related to drag, lift, turbulence dispersion, virtual mass and wall lubrication were considered in their study.

When the separated conservation equations are summed up together the Mixture model is obtained, in which only one momentum equation is calculated for both phases. The relative velocity between them is accounted through a slip model, such as Drift Flux model. In his PhD thesis, Lima [16] developed a one-dimensional code applying the Mixture model allied to phenomenological considerations in order to simulate isothermal separated, dispersed and intermittent gas-liquid flows. Pressure gradients and flow pattern transitions were verified against own experimental data.

In the particular approximation where there is no slip, the Homogeneous model is obtained. The Volume of Fluid (VOF) rests in this class. Although based on the Homogeneous model, the application of adequate advection schemes for the transport equation of volume fraction allows the VOF model to capture a separation of the phases (immiscible fluids and discernible interface) and to adequately calculate velocity fields even for counter-current situations, $e . g$. the falling film around Taylor bubbles. This approach has been extensively applied in the study of two-phase flows.

Gao et al. [17] simulated water-oil liquid-liquid stratified flows using VOF within a range of volumetric fraction and mass flow rates. Using the results, the authors developed correlations for pressure gradient and liquid holdup. The motion of a single Taylor bubble in stagnant and flowing liquid was calculated by Taha and Cui [18]. Hydrodynamic features such as bubble shape and length, rise velocity and wall shear stress were calculated in both 2D and 3D domains with a coordinate system attached to the bubble. Lu et al. [19] simulated stratified, wavy and slug horizontal flows of gas-oil two-phase mixtures. The calculated phase distributions are compared to visual observations obtained from own experiments.

Also by means of VOF model, de Schepper et al. [20] simulated horizontal gas-liquid flows with air-water and liquid-vapour gasoil. Bubble, stratified, wavy, plug, slug, annular and mist flows were identified by visual observations of phase distributions and compared to the Baker chart. Parvareh et al. [21] simulated horizontal and vertical air-water regimes in a $20 \mathrm{~mm}$ i.d., $4 \mathrm{~m}$ long tube. They also applied an Electrical Resistance Tomography (ERT) technique to obtain experimental visualizations of the flows; both results were then compared.

Da Riva and del Col [22] numerically studied the churn pattern in the entry region of an injector in a vertical tube, approximated by a $2 \mathrm{D}$ axisymmetric domain, also through VOF modeling. They observed the frequency of ring-type waves formation for air-water and R-134a liquid-vapour mixtures for different superficial velocities and tube diameters. They suggested a simplified model for the process of ring-type waves propagation. Ratkovich et al. [23] simulated slug flows of Newtonian and non-Newtonian fluids in a 2D axisymmetric domain at 
different superficial velocities. Results of averaged void fraction in a portion of the domain were compared to correlations of the literature and own experimental data.

In the present work, the Volume of Fluid formulation is applied to simulate air-water upward flows in a 2 in i.d. tube with three different lengths. Two sets of superficial velocities are evaluated. The influence of such parameters on the transient interaction of multiple air bubbles is investigated using fully three-dimensional domains and simulated data is quantitatively compared to experimental data and correlations. Results such as visual observations and void fractions histories are used to identify the churn/slug transition according to the process described by Taitel et al. [1]. Fast Fourier Transform technique is applied to calculate slugging frequencies, which are compared to correlations in the literature. Calculated histograms of void fraction are also compared to experimental data from the literature.

\section{Description of the transition process}

The churn/slug transition process used as reference in the present work is based on the description given by Taitel et al. [1]. Churn regime is treated as an entry region effect encountered upstream a fully developed slug flow in the case that sufficient pipe length exists. It is important to note that, as any pipe has a finite length, depending on operational conditions, churn flow may be present in the whole domain.

The authors state that in the entry region both phases are injected in a way that small packets of gas and liquid are generated. At this condition, the liquid slug is unstable and falls down the tube merging with the upstream liquid packet, approximately doubling its length. The gas bubble between the liquid slugs overtakes the downstream gas bubble, also doubling in size. This process repeats itself and, as the flow moves upward, the length of both liquid slug and gas bubble increases until the liquid slug attain a stable length and establish a consistent bridge between two consecutive Taylor bubbles. The decay of the velocity profile in the liquid slug is used to determine the stable liquid slug length which is suggested as $16 D$, being $D$ the tube diameter.

Between this position and the entry region, the liquid phase alternately rises and falls, characterizing the churn region, which may take place in the whole tube as the gas flowrate increases. An expression for entrance length, $l_{E}$, was derived as:

$$
\frac{l_{E}}{D}=40.6\left(\frac{U_{s}}{\sqrt{g D}}+0.22\right)
$$

where $U_{s}$ and $g$ are respectively the mixture superficial velocity and the gravitational acceleration. Equation (1) is recommended by the authors as the transition boundary between churn and slug flow regimes. In this work, this transition process was studied by means of Computational Fluid Dynamics. 


\section{Multiphase flow modeling}

The Volume of Fluid formulation, derived by Hirt and Nichols [24], is based on a scalar indicator function with values between 0 and 1 to distinguish between different fluids, which are considered as immiscible ones. Its application is recommended in situations where the interface to be captured is much larger than the mesh cell; around $4-9$ cells are required to compose a bubble. Hence, small bubbles such as the ones present in bubble flows are not suitable to be represented by VOF method unless a very fine computational grid is used.

In a two-phase mixture computation, a value of zero indicates presence of one phase and a unity value represents the other phase. The values between them indicate the existence of an interface and is a measure of the relative concentration of the fluids. In general, the approach of applying volume fractions is computationally more economical than using markers following the interface since only one value is assigned to each computational cell [25]. Other advantage is that only one conservation equation is required to be solved for the propagation of the volume fractions throughout the domain, in addition to the mass and momentum conservation equations.

\subsection{Governing equations}

In the VOF method, all the fluids share a single set of governing equations. The conservation equations are given as:

$$
\text { Mass: } \frac{\partial \rho}{\partial t}+\nabla \cdot(\rho \mathbf{U})=0
$$

where $\rho$ is the mixture density and $\mathbf{U}$ is the mixture velocity vector.

$$
\text { Momentum: } \frac{\partial(\rho \mathbf{U})}{\partial t}+\nabla \cdot(\rho \mathbf{U U})=-\nabla p+\nabla \cdot \underline{\mathbf{T}}+\rho \mathbf{g}+\mathbf{F}_{\sigma}
$$

In Eq. (3), first term on the left hand side (LHS) represents rate of increase in momentum per unit volume while the second term represents change in momentum due to convection. First term on the right hand side (RHS) is the pressure gradient; second term represents viscous and turbulent contribution; third term is the gravitational force per unit volume and last term represents the force due to surface tension, modeled by the Continuum Surface Force formulation proposed by Brackbill et al. [26].

For the cells lying at the interface, the mixture density $\rho$ and dynamic viscosity $\mu$ are related to the volume fraction and individual properties of phase $k$ as:

$$
\rho=\sum \alpha_{k} \rho_{k}, \quad \mu=\frac{\sum \alpha_{k} \rho_{k} \mu_{k}}{\sum \alpha_{k} \rho_{k}}
$$

Mixture velocity is defined as a combination of phase-weighted and massweighted variables: 


$$
\mathbf{U}=\frac{\sum \alpha_{k} \rho_{k} \mathbf{U}_{k}}{\sum \alpha_{k} \rho_{k}}
$$

For each additional phase $k$ present in the mixture, an additional transport equation for its volume fraction needs to be included in the calculation:

$$
\frac{\partial\left(\rho \alpha_{k}\right)}{\partial t}+\nabla \cdot\left(\rho \mathbf{U} \alpha_{k}\right)=S_{k}
$$

First term on LHS is the accumulation while second term represents convection. The term on RHS is the sum of sources and will considered equal to zero. Equation (6) will be solved for $N-1$ phases, respecting the constraint $\sum \alpha_{k}=1$.

One drawback of the VOF method is that, while solving the volume fraction advection equation, special interpolation schemes are required for preserving accurate results in the mass conservation equation. Conventional advection schemes, such as Second-order Upwind and QUICK, smear the step profile of the interface over several computational cells due to numerical diffusion [25]. To overcome this situation, special methods such as CICSAM [27] or line techniques are needed. In this study, the Piecewise Linear Interface Construction (PLIC) algorithm, a line technique first proposed by Youngs [28], was applied due to its ability to render a sharp and clear interface without numerical diffusion [25]. This scheme is briefly presented next.

\subsection{Near-interface treatment}

Through the PLIC algorithm, interfaces are reconstructed by a sequence of oblique or piecewise line segments which may not be continuous but build a discontinuous chain with small discontinuities. The slope of the segment is determined by the interface normal. The determination of the normal vector constitutes the first step of the reconstruction algorithm, which usually is obtained from the volume fraction gradients. The second part is related to the placement of the line segment in a way such that the volume of the resultant polygon formed by the segment and cell faces is equal to the volume fraction of the mesh cell under evaluation. From this polygon, fluid mass fluxes are then calculated through the cell sides. Finally, the volume fraction in the cell is updated from the balance of fluid fluxes calculated in the previous step. Figure 1 illustrates an example of interface reconstructed by the PLIC method.

By means of the PLIC scheme, volume fraction fields physically bounded by the constraints of values between $0-1$ can be obtained while capturing a sharp interface, essential for getting adequate results from the VOF method.

\section{Simulations}

The VOF method allied to the PLIC interpolation scheme was used to simulate incompressible air-water upward flows in a $D=2 \mathrm{in}$ i.d. tube. In order to evaluate the churn/slug transition dynamics, simulations were carried out with 

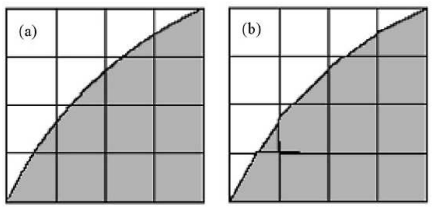

Figure 1: Interface reconstruction: (a) actual interface shape, (b) interface reconstructed through PLIC algorithm [20].

three different pipe lengths, namely $20 \mathrm{D}, 50 \mathrm{D}$ and $100 \mathrm{D}$. Air and water enters the domain through separate inlets of a mixer positioned upstream the straight pipe. The three-dimensional geometries were discretized in hexahedral cells with prismatic elements close to wall to ensure correct capturing of near-wall gradients. Largest mesh is constituted by around 363,500 cells. Details of the geometry and the mesh can be observed in Fig. 2.

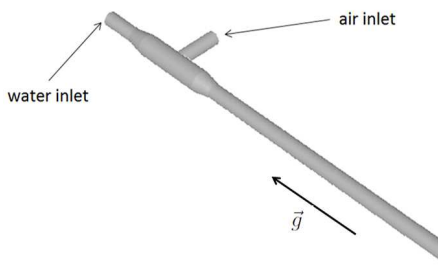

(a)

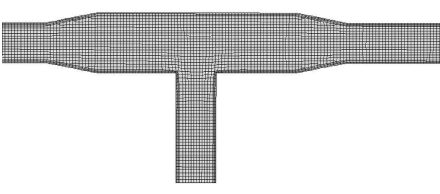

(b)

Figure 2: Details of: (a) mixer with tube, (b) hexahedral mesh cells.

Turbulence was considered by means of the Realizable $k-\epsilon$ model proposed by Shih et al. [29]. Pressure-velocity coupling was calculated via PISO algorithm [30]. Pressure values were interpolated by means of a staggered grid method [31]. As mentioned before, volume fraction is calculated with PLIC algorithm. All other variables were interpolated with Second-order Upwind method [31].

Superficial velocity of water, $U_{s l}$, was kept constant at $0.05 \mathrm{~m} / \mathrm{s}$ while air superficial velocities, $U_{s g}$, were $0.2 \mathrm{~m} / \mathrm{s}$ and $0.5 \mathrm{~m} / \mathrm{s}$. Atmospheric pressure is considered at the tube outlet. Wall functions are used to provide turbulence boundary conditions at smooth walls which experience the no slip condition.

Single-phase steady state simulations were performed with water to obtain fully developed pressure and velocities fields prior to conducting transient simulations. Air was then injected through its specific inlet. Transient simulations were carried 
out with a time-step of 0.001 seconds during 60.0 seconds, enough time to capture the transient flow development and to get statistically significant data. Fluid properties were assumed as shown in Table 1. Surface tension coefficient was specified as $0.07 \mathrm{~N} / \mathrm{m}$. Gravitational force was taken into account with acceleration $g=9.81 \mathrm{~m} / \mathrm{s}^{2}$.

Table 1: Fluid properties.

\begin{tabular}{|c||c|c|}
\hline & Water & Air \\
\hline$\rho\left[\mathrm{kg} / \mathrm{m}^{3}\right]$ & 998.2 & 1.225 \\
\hline$\mu[$ Pa.s $]$ & $1.0 e-3$ & $1.79 e-5$ \\
\hline
\end{tabular}

\section{Results}

For the superficial velocity set of $U_{s l}=0.05 \mathrm{~m} / \mathrm{s}$ and $U_{s g}=0.5 \mathrm{~m} / \mathrm{s}$, simulations were carried out with the three different tube lengths. Visualizations of phase distribution can be observed in Fig. 3 with highly distorted interfaces being captured. Bullet-shaped bubbles are identified in all the cases with larger bubbles being distinguishable as the flow moves upward, showing the ability of VOF method to capture coalescence-related phenomenon. This behaviour is in accordance with Taitel et al. observations [1].

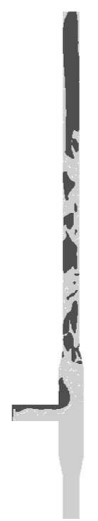

(a)

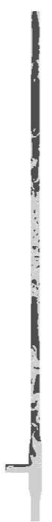

(b)

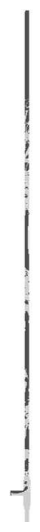

(c)

Figure 3: Phase distribution after 10 seconds with different tube lengths: (a) $20 \mathrm{D}$, (b) $50 \mathrm{D}$ and (c) $100 \mathrm{D}-U_{s l}=0.05 \mathrm{~m} / \mathrm{s}$ and $U_{s g}=0.5 \mathrm{~m} / \mathrm{s}$ - dark colour represents $100 \%$ air. 
Figure 4 shows the nose region of a Taylor bubble, where it is possible to note the liquid flow reversion and the falling film around the gas packet. With the $20 \mathrm{D}$ and $50 \mathrm{D}$ geometries, liquid slugs with stable lengths, i. e., with around $16 \mathrm{D}$, are not present; results obtained with the $100 \mathrm{D}$ domain showed the presence of such structures in the upper region of the duct.

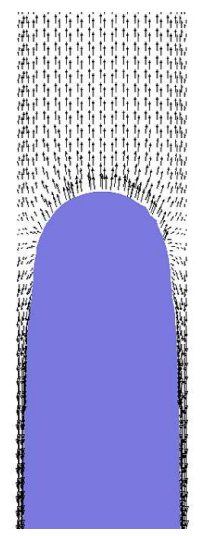

Figure 4: Velocity vectors around the nose of a Taylor bubble.

According to the churn/slug transition adopted in the present work, flows in the $20 D$ and $50 D$ tubes may be classified as churn occupying the whole domain. Both churn and slug regimes coexist in the $100 \mathrm{D}$ case. In order to objectively identify the transition point between the patterns, void fraction histories were recorded at points spaced 15 in apart along the tube centerline. Fast Fourier Transform (FFT) analyses were performed to evaluate the slugging frequency at these points. A constant frequency of $0.376 \mathrm{~Hz}$ from the point located at $67.5 \mathrm{D}$ on is noticed. This were respectively considered the simulated slugging frequency and entrance length. This procedure was also conducted for the superficial velocity set of $U_{s l}=0.05 \mathrm{~m} / \mathrm{s}$ and $U_{s g}=0.2 \mathrm{~m} / \mathrm{s}$ with the $100 \mathrm{D}$ tube geometry. A frequency of $0.579 \mathrm{~Hz}$ and an entrance length of $60.0 \mathrm{D}$ were obtained. The results of slugging frequency were compared to correlations suggested by van den Akker et al. [9], Hernandez-Perez et al. [14] and Zabaras (apud. [16]) which are summarized in Table 2.

Simulated frequencies are overestimated when compared to both van den Akker and Hernandez-Perez correlations, presenting a relative difference as high as $133 \%$. Best agreement was found to be around $24 \%$. This last correlation seems to be quite insensitive to gas superficial velocity. Against Zabaras expression, simulations provided an underestimation of frequency, with best agreement being within a $2 \%$ margin. In accordance with experimental observations [12], simulation results showed a decay of frequency as flows moves upward, observed in Fig. 5. 
Table 2: Results of slugging frequency calculated with correlations $[\mathrm{Hz}]-U_{s l}=$ $0.05 \mathrm{~m} / \mathrm{s}$.

\begin{tabular}{|l||c|c|c|}
\hline & van den Akker & Hernandez-Perez & Zabaras \\
\hline$U_{s g}=0.2 \mathrm{~m} / \mathrm{s}$ & 0.466 & 0.248 & 0.976 \\
\hline$U_{s g}=0.5 \mathrm{~m} / \mathrm{s}$ & 0.215 & 0.204 & 0.384 \\
\hline
\end{tabular}

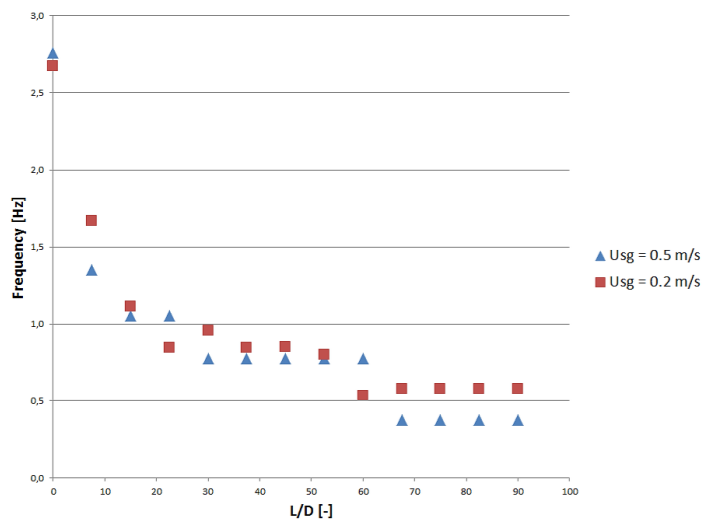

Figure 5: Decay of slugging frequency along tube centerline $-L=100 \mathrm{D}, U_{s l}=$ $0.05 \mathrm{~m} / \mathrm{s}$.

Calculated entrance lengths are around $66 \%$ and $157 \%$ higher for $U_{s g}=$ $0.5 \mathrm{~m} / \mathrm{s}$ and $U_{s g}=0.2 \mathrm{~m} / \mathrm{s}$, respectively, when compared to equation (1). Histograms of void fraction at cross sections located at $0 D, 45 D$ and $90 D$ positions were also evaluated and are shown in figure 6 . Results clearly show, as flow moves upward, the appearance of two main peaks: one at low and other at high void fraction values which are in good agreement with experimental observations. The high void fraction peak is related to the gas packets (Taylor bubbles), while the low void fraction peak is related to the satellite bubbles located in the liquid slug $[3,8]$.

\section{Concluding remarks}

Transient simulations of slug flows at two different superficial velocity sets were conducted. Three different pipe lengths were investigated in order to evaluate twophase topological structures developed in each domain. It was found that for the highest gas superficial velocity, only churn flow is observable in the $20 \mathrm{D}$ and $50 \mathrm{D}$ geometries. With the $100 \mathrm{D}$ domain, stable liquid slugs were observed with churn and slug flows coexisting in the tube. 


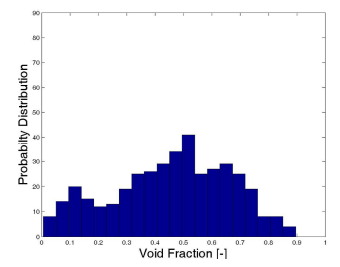

(a)

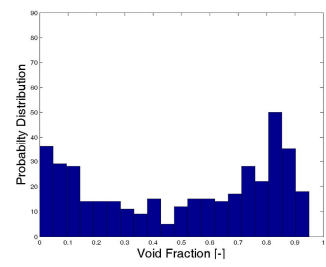

(b)

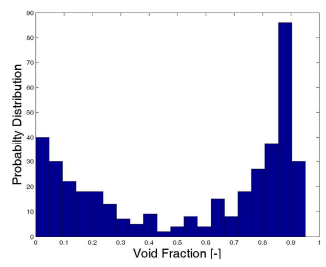

(c)

Figure 6: Histogram of void fraction at: (a) $0 \mathrm{D}$, (b) $45 \mathrm{D}$ and (c) $90 \mathrm{D}$ position $U_{s l}=0.05 \mathrm{~m} / \mathrm{s}$ and $U_{s g}=0.5 \mathrm{~m} / \mathrm{s}$.

Values of slugging frequencies and entrance lengths were in general overestimated when compared to correlations available in the literature. This behaviour may be explained by the asymmetric entrance condition, requiring longer tube lengths to achieve rotational symmetry. Another hypothesis that may contribute to the discrepancies is the gas incompressibility; in an upward flow, gas bubbles expand and accelerate due to pressure drop. Hence, the constant density simplification directly interferes in bubble dynamics. Histograms of void fractions are consistent with experimental observations showing two peaks at low and high values of void fractions at the upper region of the duct, which is characteristic of a developed slug flow.

The obtained results reveal the capability of the Volume of Fluid method in handling complex two-phase flow interactions. It is worth mentioning that the application of PLIC interface reconstruction method is essential to adequately determine the location and geometry of the interface and therefore provide meaningful results.

\section{Acknowledgements}

This work was granted by Petróleo Brasileiro S.A. (Petrobras). The authors wish to thank to Agência Nacional do Petróleo (ANP) and Conselho Nacional de Desenvolvimento Científico e Tecnológico (CNPq).

\section{References}

[1] Taitel, Y., Barnea, D. \& Dukler, A.E., Modelling flow pattern transitions for steady upward gas-liquid flow in vertical tubes. AIChE Journal, 26, pp. 345354, 1980.

[2] Spedding, P.L., Woods, G.S., Raghunathan, R.S. \& Watterson, J.K., Vertical two-phase flow. Trans IChemE, 76, pp. 612-619, 1998.

[3] Watson, M. \& Hewitt, G., Pressure effects on the slug to churn transition. International Journal of Multiphase Flow, 25, pp. 1225-1241, 1999. 
[4] Mishima, K. \& Ishii, M., Flow regime transition criteria for upward twophase flow in vertical tubes. International Journal of Heat and Mass Transfer, 27, pp. 723-737, 1984.

[5] Brauner, N. \& Barnea, D., Slug/churn transition in upward gas-liquid flow. Chemical Engineering Science, 41, pp. 159-163, 1986.

[6] Bendiksen, On the motion of long bubbles in vertical tubes. International Journal of Multiphase Flow, 11, pp. 797-812, 1985.

[7] Barnea, D., Effect of bubble shape on pressure drop calculations in vertical slug flow. International Journal of Multiphase Flow, 16, pp. 79-89, 1990.

[8] Jones Jr., O.C., The interrelation between void fraction fluctuations and flow patterns in two-phase flow. International Journal of Multiphase Flow, 2, pp. 273-306, 1975.

[9] van den Akker, H.E.A., Legius, H.J.W.M. \& Narumo, T., Measurements on wave propagation and bubble and slug velocities in concurrent upward twophase flow. Experimental Thermal and Fluid Science, 15, pp. 267-278, 1997.

[10] Heywood, N.I. \& Richardson, J.F., Slug flow of air-water mixtures in a horizontal pipe: Determination of liquid holdup by gamma-ray absorption. Chemical Engineering Science, 34, pp. 17-30, 1979.

[11] Polonsky, S., Barnea, D. \& Shemer, L., Averaged and time-dependent characteristics of the motion of an elongated bubble in a vertical pipe. International Journal of Multiphase Flow, 25, pp. 795-812, 1999.

[12] van Hout, R., Shemer, L. \& Barnea, D., Evolution of hydrodynamic and statistical parameters of gas-liquid slug flow along inclined pipes. Chemical Engineering Science, 58, pp. 115-133, 2003.

[13] Shemer, L., Hydrodynamic and statistical parameters of slug flow. International Journal of Heat and Fluid Flow, 24, pp. 334-344, 2003.

[14] Hernandez-Perez, V., Abdulkadir, M. \& Azzopardi, J., B., Slugging frequency correlation for inclined gas-liquid flow. World Academy of Science, Engineering and Technology, 37, pp. 44-51, 2010.

[15] Ekambara, K., Sanders, R.S., Nandakumar, K. \& Masliyah, J.H., Cfd simulation of bubbly two-phase flow in horizontal pipes. Chemical Engineering Journal, 144, pp. 277-288, 2008.

[16] Lima, L.E.M., Analise do modelo de Mistura aplicado em escoamentos isotermicos gas-liquido. Ph.D. thesis, Universidade Estadual de Campinas, 2011.

[17] Gao, H., Gu, H. \& Guo, L., Numerical study of stratified oil-water two-phase turbulent flow in a horizontal tube. International Journal of Heat and Mass Transfer, 46, pp. 749-754, 2003.

[18] Taha, T. \& Cui, Z., Cfd modelling of slug flow in vertical tubes. Chemical Engineering Science, 61, pp. 676-687, 2006.

[19] Lu, G., Wang, J. \& Jia, Z., Experimental and numerical investigations on horizontal oil-gas flow. Journal of Hydrodynamics Ser B, 19 (6), p. 19 (6), 2007. 
[20] de Schepper, S.C.K., Heynderickx, G.J. \& Marin, G.B., Cfd modeling of all gas-liquid and vapor-liquid flow regimes predicted by the baker chart. Chemical Engineering Journal, 138, pp. 349-357, 2008.

[21] Parvareh, A., Rahimi, M., Alizadehdakhel, A. \& Alsairafi, A.A., Cfd and ert investigations on two-phase flow regimes in vertical and horizontal tubes. International Communications in Heat and Mass Transfer, 37, pp. 304-311, 2010.

[22] da Riva, E. \& del Col, D., Numerical simulation of churn flow in a vertical pipe. Chemical Engineering Science, 64, pp. 3753-3765, 2009.

[23] Ratkovich, N., Majumder, S. \& Bentzen, T., Empirical correlations and cfd simulations of vertical two-phase gasliquid (newtonian and non-newtonian) slug flow compared against experimental data of void fraction. Chemical Engineering Research and Design, 2012.

[24] Hirt, C.W. \& Nichols, B.D., Volume of fluid (vof) method for dynamics of free surfaces. Journal of Computational Physics, 39, pp. 201-225, 1981.

[25] Yeoh, G.H. \& Tu, J., Computational techniques for multi-phase flows. Elsevier, 2010.

[26] Brackbill, J.U., Kothe, D.B. \& Zemach, C., A continuum method for modeling surface tension. Journal of Computational Physics, 100, pp. 335354, 1992.

[27] Ubbink, O., Numerical prediction of two fluids system with sharp interfaces. Tese de doutorado, Department of Mechanical Engineering - Imperial College of Science, Technology \& Medicine, 1997.

[28] Youngs, D.L., Numerical methods for fluid dynamics, Academic Press, chapter Time-dependent multi-material flow with large fluid distortion, pp. 273-285, 1982.

[29] Shih, T.H., Liou, W.W., Shabbir, A., Yang, Z. \& Zhu, J., A new k-epsilon eddy-viscosity model for high Reynolds number turbulent flows. Computers Fluids, 24, pp. 227-238, 1995.

[30] Versteeg, H.K. \& Malalasekera, W., An introduction to computational fluid dynamics - The finite volume method. Longman Scientific \& Technical, 1995.

[31] Ferziger, J.H. \& Peric, M., Computational methods for fluid dynamics. Springer, 2002. 